\title{
El movimiento médico y el doctor Ismael Cosío Villegas. A medio siglo
}

\author{
José Luis Sandoval-Gutiérrez
}

Secretaría de Salud, Instituto Nacional de Enfermedades Respiratorias, Servicio de Neumología, Ciudad de México, México

\begin{abstract}
Resumen
En 1964, los médicos residentes e internos de la República Mexicana decidieron realizar un paro de labores que se extendió por meses; varios médicos notables decidieron unirse a las causas que motivaron dicho movimiento, entre ellos el director general en ese momento del Sanatorio para Tuberculosos de Huipulco, el doctor Ismael Cosío Villegas, cuya participación y entrega es histórica. La determinación del doctor Cosío Villegas provocó que se le exigiera su renuncia. La comunidad médica aún se desconoce su trayectoria.
\end{abstract}

PALABRAS CLAVE: Historia de la medicina. Movimiento médico. Ismael Cosío Villegas. Instituto Nacional de Enfermedades Respiratorias. Examen nacional de residencias médicas.

\begin{abstract}
In 1964, medical residents and interns of the Mexican Republic decided to car ry out a work stoppage that stretched over for several months. Several prominent doctors decided to join the causes that motivated the movement, among which Dr. Ismael Cosío Villegas, who at that moment was the director general of the Huipulco hospital for tuberculosis patients; his participation and dedication are historical, and his determination caused for his resignation to be demanded. His trajectory remains practically unknown by the medical community.
\end{abstract}

KEY WORDS: History of medicine. Medical movement. Ismael Cosío Villegas. Instituto Nacional de Enfermedades Respiratorias. National exam for medical residency.

A finales de 1964, en el Hospital 20 de Noviembre del Instituto de Seguridad y Servicios Sociales de los Trabajadores del Estado (ISSSTE), actualmente Centro Médico Nacional 20 de Noviembre, en la Ciudad de México, se forjó una manifestación de médicos internos y residentes provocada por la suspensión del pago correspondiente al aguinaldo de fin de año, aunada a repetidas desatenciones al cuerpo médico, lo que motivó que se declararan en huelga, ${ }^{1}$ que consistió en guardias mínimas y solo atención a pacientes con situación médica o quirúrgica de urgencia.

En poco tiempo se sumaron hospitales de diferentes sistemas de salud, como el Hospital General de México, Hospital Juárez, Hospital Rubén Leñero y algunos hospitales de los ahora considerados Institutos Nacionales de Salud; posteriormente se agregaron centros médicos de Guadalajara y Monterrey. Al final, diversos nosocomios de otros estados se sumaron a este levantamiento pacifico, que por ser encabezado por personal de salud fue denominado Movimiento blanco. ${ }^{2}$

Como manifestación principal se realizó la "marcha del silencio" (Figura 1), donde el contingente médico, apoyado por personal de enfermería, recorrió las calles del centro de la Ciudad de México, llevando pancartas y letreros en los que se exponía demandas, peticiones y consignas; numerosos participantes del contingente caminaban con los labios sellados con material de
Correspondencia:

José Luis Sandoval-Gutiérrez

E-mail: sandovalgutierrez@gmail.com
Fecha de recepción: 14-07-2015

Fecha de aceptación: 15-07-2015

DOI://dx.doi.org/10.24875/GMM.18001792
Gac Med Mex. 2018;154:254-257

Disponible en PubMed

www.gacetamedicademexico.com 
curación. Se hicieron formaciones en la explanada del zócalo de la ciudad. La mayoría de los espectadores mostraba solidaridad con el movimiento, pero también quienes insultaban al grupo, incluso, hubo presencia de provocadores. Era una época poco tolerante con este tipo de expresiones; el presidente en turno de México no mostró simpatía con la comisión representante.

La negativa al diálogo dio como consecuencia que el movimiento se extendiera por varios meses. Se integró la Alianza Médica Nacional, que organizaba las reuniones y los debates sobre la situación y la cual desafortunadamente desapareció después del movimiento. Hasta el momento no han existido voces que muestren voluntad de reorganización civil de una asociación médica nacional que agremie a los facultativos del país, independientemente de su especialidad o formación clínica, como sucede en otros países. ${ }^{3}$

Pocos medios de comunicación dieron cobertura a la situación que se vivía y al hacerlo, en no pocas ocasiones, era para desprestigiar al movimiento.

Médicos y enfermeras fueron desalojados por la fuerza de los centros hospitalarios, incluso con grupos de fuerza de reacción física ("granaderos") en el Hospital 20 de Noviembre, donde se inició el movimiento (Figura 2).

Ha pasado medio siglo desde esta expresión gremial, durante la cual varios de sus líderes fueron cesados, entre ellos el doctor Ismael Cosío Villegas, neumólogo y cirujano de tórax, en ese momento director general del Sanatorio para Tuberculosos de Huipulco, actualmente Instituto Nacional de Enfermedades Respiratorias Ismael Cosío Villegas, INER (Figura 3). La negativa del doctor Cosío Villegas a dejar de apoyar a los médicos que estaban en el movimiento ocasionó que se le exigiera su renuncia, la cual cumplió cabalmente el 15 de enero de $1965 .{ }^{4}$ En su carta de renuncia al consejo técnico expresó lo siguiente:

Lamento separarme de este sanatorio al que he servido con lealtad, cariño y perseverancia durante 28 años, pero no quiero que mis colegas, alumnos y estudiantes pierdan la fe y la confianza depositada en mi modesta personalidad, que he procurado forjarla en el desinterés, el valor civil y el más alto espíritu de compañerismo.

\section{En su misiva a la Secretaría de Salud escribió:}

No estoy resentido con nadie, sobre todo estoy tranquilo y de acuerdo con mi conducta, diga al ministro que este documento no me sorprende porque lo esperaba, dígale también que salir de Huipulco, bajo estas circunstancias, para mí es un timbre de orgullo.

El doctor Cosío Villegas se retiró al ámbito académico y a su práctica privada, fue nombrado profesor



Figura 1. Manifestación del Movimiento blanco en la Ciudad de México, 1964. Imagen obtenida de http://www.kehagoaqui.com.

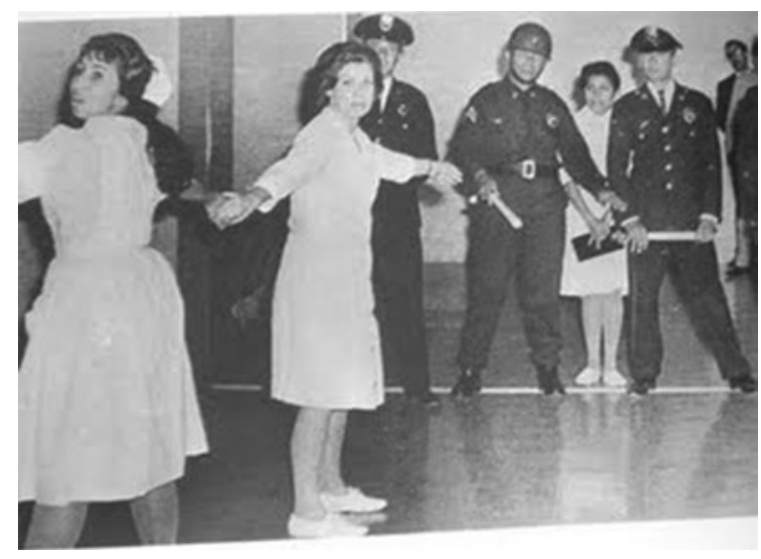

Figura 2. Apoyo del personal de enfermería al gremio médico para evitar el desalojo del Hospital 20 de Noviembre (ISSSTE). Imagen obtenida de https://enlacecursoshistoria.files.wordpress.com/2009/11/ el-movimiento-medico-004.jpg.



Figura 3. Imagen del Instituto Nacional de Enfermedades Respiratorias Ismael Cosío Villegas; en la entrada y plaza principal se observa el busto del maestro. Imagen obtenida de http://www.iner.salud.gob.mx/ media/70152/english_vhome.jpg. 
emérito por la Universidad Nacional Autónoma de México (UNAM) y doctor honoris causa por la Universidad Autónoma de Puebla, pero nunca volvió a su casa, el hoy Instituto Nacional de Enfermedades Respiratorias, que desde el 2006 lleva su nombre (Figura 4).

En 1977 se le realizó un homenaje por sus 50 años de trayectoria en el Instituto Nacional de Cardiología (Figura 5), con la presencia del doctor Ignacio Chávez y representantes de la Academia Nacional de Medicina, de la cual el doctor Cosío Villegas fue presidente en 1961. En esa ocasión, el doctor Chávez expresó:

Llega a la vejez con el orgullo de su vida limpia, laboriosa y fecunda, dejando tras de sí su obra como un legado a las generaciones médicas que siguen. [...]

El hombre que venimos a honrar es sobre todo un profesor nato. Sus discípulos le siguen y le admiran y esto prueba que en él encuentran dones de sabiduría. Pero hay algo más, lo siguen y eso prueba que encuentran en él dones de generosidad. [...]

Con ceguera, el país condenaba así al retiro a uno de sus altos valores en la enseñanza, la vida no le ha torcido el alma como a otros. [...]

El tiempo le ha dado la razón moral e ideológica de la lucha encabezada por él hace medio siglo.

Durante la ceremonia de la inclusión del nombre del doctor Ismael Cosío Villegas al INER, el doctor Raúl Cicero mencionó:

Cosío Villegas en los últimos años fue objeto de débiles homenajes por diversos grupos. El maestro murió el 2 de agosto de 1985; pero la realidad es que no se ha reconocido cabalmente su valor humano, fue congruente en el pensamiento y en la acción, ni su labor como maestro de la neumología mexicana. En el Hospital General de México, de donde salió Cosío Villegas a fundar este honorable Instituto, aprendimos a respetarlo y a quererlo. Alejandro Celis, Carlos Pacheco, Octavio Rivero, Raúl Cicero, José Kuthy, Hermilo Esquivel Medina, entre otros - nuestros maestrosasí nos enseñaron a respetar a Cosío Villegas.

En una ocasión, hace 54 años, el maestro Cosío en una sesión de la Academia mencionó:

\begin{abstract}
El hombre social tiene necesidad de justicia, lograda solamente con la desaparición de la inmoralidad pública, en esta justicia está el plan revolucionario de nuestros tiempos: mejor distribución de la riqueza, paridad en derechos y obligaciones, respeto a los valores humanos, estas son cuestiones de fisiología social.
\end{abstract}

Mucho del saber y pensamiento del maestro lo compartía con grandes personalidades de la época: Diego Rivera, Frida Khalo, Siqueiros, José Gaos, Gabriela Mistral, Pablo Neruda, Guadalupe Amor, Narciso Bassols; y con otros médicos: Salvador Zubirán, Federico Gómez, Isaac Costero, Rosenblueth, Gustavo Baz, Ignacio Chávez, por citar algunos, quienes



Figura 4. Doctor Ismael Cosío Villegas. Imagen obtenida de http:// www.facmed.unam.mx/sms/seam2k1/2008/oct_04_ponencia.html.

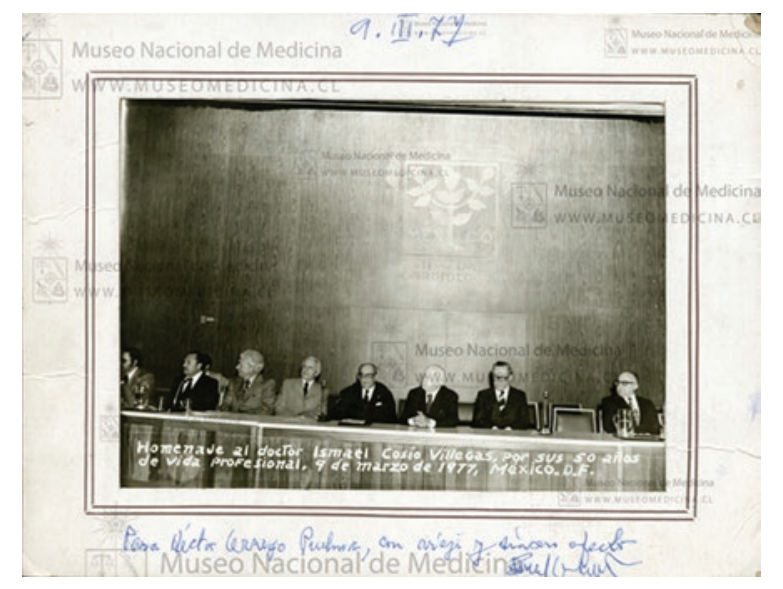

Figura 5. El doctor Ismael Cosío Villegas, en el tercer sitio de derecha a izquierda; el doctor Ignacio Chávez en el cuarto. Imagen obtenida de http://bibliotecadigital.uchile.cl/client/search/asset/57936.

reconocían las capacidades y el liderazgo del doctor Cosío Villegas, lo que indica su gran calidad intelectual.

Hay mucho que analizar del Movimiento blanco: fue el precedente para que las residencias médicas hoy gocen de registro y reconocimiento adecuados, se realice un examen nacional de aspirantes a residencias médicas (EXARM), ${ }^{5}$ que los médicos seleccionados tengan el derecho a una beca, a una formación regida por un plan académico con reconocimiento institucional y universitario, y que los especialistas sean certificados por su respectivo consejo y obtengan una cédula profesional.

El doctor Norberto Treviño, uno de los más destacados líderes del movimiento, expresó en alguna ocasión:

El movimiento médico constituyó el más generalizado y vigoroso intento de alcanzar la unidad médica, el resultado 
fue la división, el silencio del gremio médico. Hoy a cuarenta años de ese movimiento, aquel esfuerzo no fue en vano, al médico se le reconoce, se le consideran sus legítimas características de trabajador, sus problemas económico-sociales, su seguridad de hombre y de profesionista.

Lo que ahora se da por hecho, hace 50 años no era realidad. En la actualidad es increíble que la comunidad médica y aun los recién egresados de la licenciatura conozcan a la perfección los movimientos estudiantiles de 1968 y 1971, incluso el ferrocarrilero, pero desconozcan el Movimiento blanco, anterior a los mencionados.

Como fenómenos herederos de 1964 se puede mencionar que en 2014 se organizaron dos movimientos:

- Yo soy 17, iniciado por médicos de todo el país para apoyar a 16 facultativos de Guadalajara acusados de homicidio por el fallecimiento de un paciente con enfermedad pulmonar crítica; el padre del adolescente, abogado, trató de culpar al grupo que atendió a su hijo.

Al parecer las demandas del gremio aún no han sido satisfechas y el espíritu del Movimiento blanco se aparece ocasionalmente.

- El otro movimiento fue organizado por enfermeras de todo el país que lucharon por sus derechos laborales e hicieron un plantón afuera de la Secretaría de Salud para impedir el cambio de categoría en sus centros de trabajo.

Hace falta la revisión histórica y el intercambio de ideas sobre el Movimiento blanco, que empoderó a las especialidades médicas, pero que reprimió a sus dirigentes y castigó a sus líderes, entre ellos al pilar de la neumología mexicana, el doctor Ismael Cosío Villegas.

En 1989, el doctor Carlos Pacheco mencionaba:

Cosío Villegas conocía a los hombres y los catalogaba adecuadamente. Era duro con quien lo merecía, pero nunca se ensañaba con el caído ni molestaba al débil, fue orgulloso. Hombre de ideas políticas firmes. Fue capaz de renunciar a su gran carrera en el momento cumbre para recluirse en el ostracismo, como una protesta muda en defensa de sus convicciones y en afrenta de quienes tan injustamente procedieron en su contra.

Fernando Benítez, periodista, editor, promotor cultural, indigenista e intelectual mexicano, expresó lo siguiente:

Formó generaciones de médicos y llegado el momento luchó por la libertad y la dignidad de sus colegas. Pionero de la neumología, Ismael ha sido uno de los hombres más extraordinarios que he conocido, ha mantenido una extraña fidelidad a sus ideas en tierra de oportunistas, combatiente de la justicia, consagró su vida a defender sus convicciones más profundas.

El 4 de febrero del presente año se realizó una sesión general en el Auditorio del INER, encabezada por el doctor Jaime Villalba Caloca, pionero del trasplante pulmonar y exdirector general del Instituto, donde se conmemoró la participación del doctor Cosío Villegas en el movimiento médico.

El maestro Cosío Villegas fue congruente con su sentir, pensar y actuar. Aunque murió hace 30 años, es necesario que su obra se difunda en las escuelas y facultades de medicina, para hacer justicia a uno de nuestros más notables personajes científicos.

Como mencionó el filósofo mexicano Antonio Caso, "igualar con la vida el pensamiento". El maestro Cosío Villegas siguió esta rectitud hasta su muerte.

\section{Bibliografía}

1. Sandoval-Gutiérrez JL. A 35 años del movimiento médico en México. Med Int Med. 2000;16(3):157-159.

2. Pozas-Horcasitas R. La democracia en blanco: el movimiento médico en México, 1964-1965. México: Siglo XXI; 1993.

3. American Medical Association. [Sitio web]. [Consultado 2015 Jul 9]. Disponible en: http://www.ama-assn.org/ama

4. Wikipedia, la enciclopedia libre [Sitio web]. Ismael Cosío Villegas. Disponible en: https://es.wikipedia.org/wiki/lsmael_Cos\%C3\%ADo_Villegas

5. Comisión Interinstitucional para la Formación de Recursos Humanos para la Salud. [Sitio web]. [Consultado 2015 Jul 8]. Disponible en: http:// www.cifrhs.salud.gob.mx 